\title{
Lipid bilayer hydrodynamic drag
}

\author{
Philip E. Jahl $\odot$ and Raghuveer Parthasarathy $\odot$ \\ Department of Physics and Materials Science Institute, University of Oregon, Eugene, Oregon 97401, USA
}

(Received 5 July 2019; accepted 9 January 2020; published 6 February 2020)

\begin{abstract}
The hydrodynamic drag at a lipid bilayer surface determines in part the flow properties of suspensions of cells and liposomes. Given the fluidity of lipid bilayers, it is not obvious a priori whether boundary conditions like those of solid objects in water, inviscid fluids in water, or something intermediate should apply at the waterbilayer interface. Though boundary conditions equivalent to those of no-slip solid-liquid interfaces have been widely assumed for decades, a direct measurement of this fundamental aspect of membrane rheology for free bilayers is lacking. We apply light sheet fluorescence microscopy to image freely diffusing phospholipid vesicles and determined the hydrodynamic drag coefficient $C \pi \eta R$, where $\eta$ is the external fluid viscosity, $R$ is the vesicle radius, and the dimensionless $C$ characterizes the surface boundary condition. We find that $C=5.92 \pm 0.13$ (stat.) \pm 0.16 (syst.), matching the theoretical value of $C=6$ for a rigid sphere and far from the $C=4$ value for a boundary with zero shear stress.
\end{abstract}

DOI: 10.1103/PhysRevResearch.2.013132

\section{INTRODUCTION}

Interfaces between lipid bilayers and aqueous solutions are present in countless environments both natural, such as at cell and organelle membranes, and artificial, such as in suspensions of liposome-encapsulated drugs. The hydrodynamics of bilayers and bilayer-bound objects are therefore of considerable interest [1-8]. In particular, the rheology of red blood cells in vivo [9] and suspensions of cells and liposomes in vitro $[10,11]$ depends directly on the nature of the flow boundary condition of the bilayer-water interface. It has been widely assumed throughout work spanning many decades that this interface is well described by the boundary condition characteristic of a rigid solid [3,12-16]. Experimental and computational studies of tracer particles flowing inside and outside lipid vesicles are consistent with this claim [17-19], but the complexity of the relationships between particle motions and membrane properties prohibit straightforward readouts. However, lipid bilayers are Newtonian fluids $[5,7,20]$, and it has been speculated that their aqueous interfaces may therefore behave more like low-shearstress boundaries or have characteristics intermediate between inviscid and solidlike extremes [21]. For bulk liquid droplets, intermediate shear conditions arise as internal fluid flows are directed in the opposite direction as drag-induced surface flows. Theoretically, therefore, an ideal spherical shell composed of an incompressible fluid would behave similarly to a rigid solid sphere, as the fluid cannot move to the interior space. The extent to which a real lipid vesicle behaves, in this sense, as an ideal spherical shell is experimentally

Published by the American Physical Society under the terms of the Creative Commons Attribution 4.0 International license. Further distribution of this work must maintain attribution to the author(s) and the published article's title, journal citation, and DOI. unclear. One could imagine, for example, that differential flows between the two leaflets of the bilayer could allow relative tank-treading motions that reduce drag from the hardsphere value. Molecular dynamics simulations often report finite shear at the bilayer-water interface (see, e.g., [4,22]) and also give estimates of interleaflet slip [22]. One could alternatively imagine that thermally driven height fluctuations, even if too small to resolve, perceptibly increase drag.

Remarkably, despite its fundamental importance and widespread applicability, we are aware of almost no measurements of the hydrodynamic boundary conditions at lipid bilayer surfaces. One study was able to use a dynamic surface force apparatus to probe lipid bilayers supported on solid surfaces, reporting no-slip boundary conditions at the bilayer-water interface, as would be the case at a solid surface in water [23]. However, the presence of a solid support is well known to alter membrane hydrodynamics, reducing lipid diffusion by roughly an order of magnitude compared to free bilayers and inhibiting large-scale spatial organization $[24,25]$. Determining the flow boundary conditions at free lipid bilayers remains an open problem.

In principle, the interfacial boundary condition of an object can be determined from measurements of its Brownian motion. For a sphere of radius $R$, the diffusion coefficient $D$ is given by the Stokes-Einstein relation

$$
D=\frac{k_{B} T}{C \pi \eta R}
$$

where $k_{B}$ is Boltzmann's constant, $T$ is the temperature, $\eta$ is the external fluid viscosity, and $C$ is a dimensionless constant that characterizes the boundary condition. It is well known that $C=6$ corresponds to the limiting case of a rigid sphere and $C=4$ corresponds to an interface with no shear stress, as is the case for a liquid sphere of zero viscosity. More generally, for a sphere of viscosity $\eta_{\text {internal }}$ in an external liquid 
of viscosity $\eta_{\text {external, }}$ with

$$
\lambda=\frac{\eta_{\text {internal }}}{\eta_{\text {external }}},
$$

the boundary constant is [26-28]

$$
C=4 \frac{3 \lambda+2}{2 \lambda+2} \text {. }
$$

We note that determining $C$ by measuring $D$ does not provide insight into local flows that may occur at length scales much smaller than the vesicle size or timescales much shorter than attainable measurement times. Rather, it provides a characterization of the average effective hydrodynamic properties of the vesicle.

In practice, determining $C$ by measuring $D$ is nontrivial due to potential hydrodynamic influence from nearby surfaces, such as container walls, and the requirement of highprecision determination of the object's positions and radius. We surmount these challenges by applying light sheet fluorescence microscopy [29-32] together with fast, accurate tracking techniques [33] to characterize the diffusive motion of spherical phospholipid vesicles. Light sheet fluorescence microscopy provides optical sectioning of three-dimensional samples, enabling the imaging of vesicles hundreds of microns (tens of vesicle diameters) away from the walls of the imaging chamber [30]. We verified our methodology by characterizing the diffusive motion of solid microspheres in an aqueous medium and water droplets in benzyl alcohol, as detailed below, which gives $C=6.28 \pm 0.15$ and $C=4.36 \pm$ 0.28 , respectively, consistent with theoretical expectations. We are therefore able to accurately measure the entire range of boundary behaviors. We then characterized lipid vesicles composed primarily of the common phospholipid 1,2dioleoyl-sn-glycero-3-phosphocholine (DOPC), determining that $C=5.92 \pm 0.13$ (stat.) \pm 0.16 (syst.). This establishes that the bilayer-water interface is well described by the interfacial shear stress condition of a solid object.

\section{METHODS}

We performed light sheet fluorescence microscopy using a home-built instrument that closely follows the design of Keller et al. [32] and is described in detail in [31]. In brief, excitation light was provided by a 488-nm laser with an output power of $50 \mathrm{~mW}$, which was scanned by a galvanometer mirror and focused by an objective lens to form a sheet in the sample chamber. The minimum thickness of the sheet was approximately equal to $3 \mu \mathrm{m}$, extending over a lateral extent (Rayleigh length) of approximately $100 \mu \mathrm{m}$. Images were captured through a $40 \times 1.0$ numerical aperture Plan-apo objective lens (Zeiss) perpendicular to the excitation plane and recorded with a 5.5 Mpixel sCMOS camera (pco.edge, Cooke Corp.). A schematic of the setup is shown in Fig. 1(a).

Suspensions of either lipid vesicles or polystyrene microspheres in $0.1 M$ sucrose, or deionized water droplets in benzyl alcohol, were placed in a square cross-section glass cuvette (Starna Cells, part No. 3-3.45-SOG-3), which was mounted to a movable translation stage and inserted in the light sheet microscopy chamber. The distance between imaged objects and the cuvette walls was several hundred micrometers.

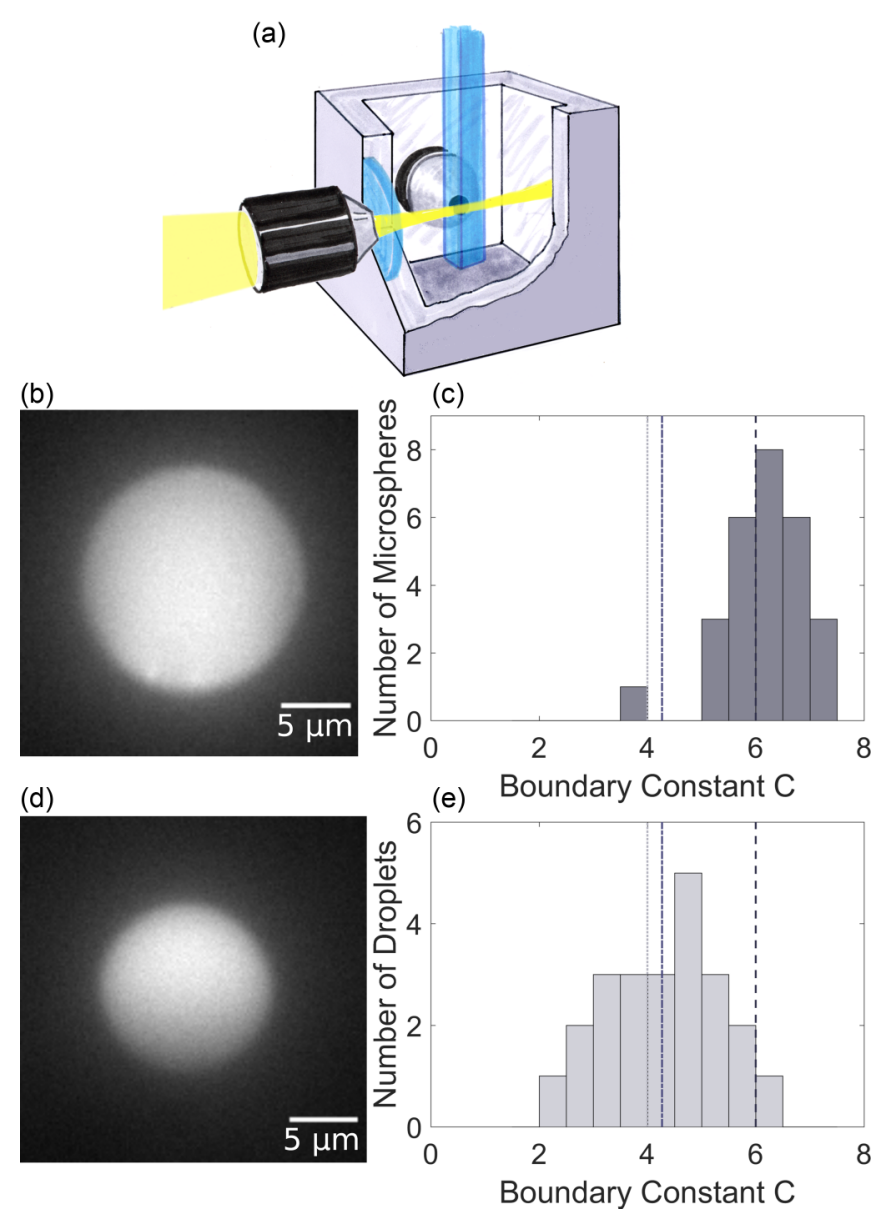

FIG. 1. (a) Schematic of the setup for light sheet fluorescence microscopy of vesicle diffusion. The excitation laser is shown entering the sample chamber from the left as it is focused into a thin sheet. Light emitted by the sample is collected by the objective lens shown behind the cuvette. (b) Typical light sheet fluorescence image of the central plane of a $15-\mu \mathrm{m}$-diam polystyrene microsphere. (c) Histogram of $C$ for 29 particles, giving a mean plus or minus standard error of $C=6.28 \pm 0.15$. (d) Typical light sheet fluorescence image of the central plane of a fluorescein-dyed water droplet in benzyl alcohol. (e) Histogram of $C$ values for 25 droplets, giving a mean plus or minus standard error of $C=4.36 \pm 0.29$. In (b) and (d) the dash-dotted line indicates the theoretical value of $C=4.27$ for water in benzyl alcohol and the dashed and dotted lines indicate the theoretical values of $C=6$ and $C=4$, respectively, for the boundary conditions of a rigid sphere and an inviscid (zero shear stress) sphere.

For beads, droplets, and vesicles the optical plane intersecting the sphere center (the "equatorial" plane) was readily evident due to a lack of out-of-focus light outside the bright ring or disk, due to the few-micron sheet thickness. This is illustrated in movie 1 in [34], which shows a three-dimensional scan through a lipid vesicle.

To assess the accuracy of our methods, we examined diffusion of objects with well known flow boundary conditions: solid polystyrene microspheres and deionized water droplets suspended in benzyl alcohol. The microspheres were fluorescein-labeled polystyrene beads of nominal diameter 
$15.45 \pm 0.70 \mu \mathrm{m}$ (mean plus or minus standard deviation; Bangs Laboratory, part No. FSDG009). Light sheet fluorescence images were captured for durations of $15 \mathrm{~s}$ at 33.33 frames/s. A typical image is shown in Fig. 1(b). For the first image, we determine the particle center using the radial symmetry-based algorithm described in [33], which provides rapid localization with accuracy close to theoretical limits. In brief, the center is calculated as the point that minimizes the total distance to lines derived from intensity gradients throughout the image. The original algorithm was modified to only weight intensity gradients from the vicinity of the bead, limiting the effects of noise outside the particle, such as fluorescent debris or light from other beads. Each remaining image was cross correlated with the previous image and the original radial symmetry-based algorithm was applied to the cross correlation to determine the shift between each frame. From the particle positions, we determine the diffusion coefficient $D$ using the covariance-based estimator described by Vestergaard et al. [35]. Not only does this provide greater accuracy than, for example, linear fits of mean-square displacements to time intervals, but it also provides robust estimates of localization accuracy and goodness of fit to a randomwalk model [35] that we make use of in assessing vesicle data below. We experimentally determined the radius of each bead by Hough transformation following edge detection [36], which produces from each input image a series of output images corresponding to each possible radius candidate, with the true object radius giving a bright, compact transform image. Across all beads, this gives an average diameter of $15.06 \pm 0.41 \mu \mathrm{m}$ (mean plus or minus standard deviation), consistent with the nominal value of $15.45 \pm 0.70 \mu \mathrm{m}$. Using the measured radius, the literature value for the viscosity of deionized water, and the ambient temperature $T=293 \mathrm{~K}$, we use Eq. (1) to determine $C$ for each microsphere. We show the histogram of $C$ values for $N=29$ microspheres in Fig. 1(b). The mean plus or minus standard error is $C=6.28 \pm 0.15$, slightly higher than but consistent with the expected $C=6$ for solid particles. (Using the nominal rather than the measured microsphere radius gives $C=6.12$, matching the theoretical value within uncertainties. The microsphere edges are less well defined than those of the vesicles; in the latter case, we note explicitly the uncertainty in $R$ below.)

The assessment of liquid droplets is similar. The droplets were deionized water dyed with $75 \mathrm{mg} / \mathrm{ml}$ fluorescein, suspended in benzyl alcohol, formed into an emulsion by vigorous shaking. Fluorescein has very low solubility in water, but nonetheless preferentially labels the aqueous phase. Benzyl alcohol was chosen because its density, $1.045 \mathrm{~g} / \mathrm{ml}$, is similar to that of water, limiting gravity-induced drift. The viscosity of benzyl alcohol is $\eta=6.29 \times 10^{-3} \mathrm{Pas}$ [37] and so the expected $C=4.27$. The droplets were imaged for $60 \mathrm{~s}$ at 8.33 frames/s. A typical image is shown in Fig. 1(c). Using the same procedures described above for center positions and radii, we determined $C$ for each water droplet. Figure 1(d) shows the histogram of $C$ values for $N=25$ droplets. The mean plus or minus standard error is $C=4.36 \pm 0.29$, consistent with the expected value. Notably, $C$ increased with time for these droplets, likely due to adsorption of fluorescein to the boundary as the poorly soluble dye lowers the oil-water interfacial energy. With sufficient adsorption, the droplet properties are those of an oil-fluorescein interface rather than an oil-water one and may reflect possible ordering of a fluorescein monolayer or pure monolayer hydrodynamics. The $C$ value stated was determined from data within $20 \mathrm{~min}$ of emulsion preparation.

The lipid vesicles we examined were composed of $94 \%$ DOPC and 6\% 1,2-dipalmitoyl-sn-glyercero-3phophoethanolamine- $N$-(7-nitro-2-1,3-benzoxadiazol-4-yl)

(NBD-PE) (Avanti Polar Lipids). Phosphatidylcholine lipids are a major constituent of cellular membranes. The head-group-conjugated NBD probe has an isotropic orientation relative to the lipid bilayer plane (unlike, for example, probes such as Texas Red [38]) and so provides vesicle images of symmetric fluorescence under polarized laser excitation. The vesicles had radii between 3.1 and $25.7 \mu \mathrm{m}$ with a mean of $12.2 \mu \mathrm{m}$ and a standard deviation of $4.0 \mu \mathrm{m}$. Vesicles were imaged for $15 \mathrm{~s}$ at 33.33 frames/s. The vesicles were created by electroformation, as in [39]. In brief, the desired lipids were dried on glass slides with an indium tin oxide coating, hydrated with a $0.1 M$ sucrose solution, and subjected to an oscillating electric field to stimulate vesicle formation. Vesicles were added to a sample cuvette containing $0.1 \mathrm{M}$ sucrose so that the interior and exterior of the vesicles would be matched in density and osmolarity. We use the literature value for the viscosity of $0.1 M$ sucrose, $\eta=1.095 \times 10^{-3}$ Pa s [40], in our analyses. Low levels of drift were present in the experiments, possibly due to convection and imperfect density matching. We therefore subtracted the best-fit linear trajectory, i.e., constant velocity, from each vesicle trajectory and used only the horizontal component of trajectories. Rare frame-to-frame displacements more than three standard deviations from the mean (less than $0.5 \%$ of frames) appeared to indicate large-scale instrument vibrations, and trajectories were analyzed piecewise around such points.

We assessed the accuracy of center- and radius-finding algorithms for vesicle images by applying them to simulated images of bright rings with a range of radii and signal-to-noise ratios (SNRs), mimicking the form of the vesicle images, as in Ref. [30] and similar to the ring images in Ref. [33]. In brief, a high-resolution image of a thin annulus was convolved with the detection point-spread function (PSF), pixelated, and subjected to Poisson-distributed noise. We used the theoretical PSF of the emission wavelength and numerical aperture, which has a full width at half maximum of $0.19 \mu \mathrm{m}$. Because of the occasional presence of lipid matter in and around the vesicle edge, the radial center-finding algorithm is weighted with a hyperbolic tangent function centered around the ring of the vesicle so as to only use the intensity gradient from the edge of the vesicle. From analysis of simulated images, the radial-symmetry-based localization gives an estimated localization error of approximately $3 \mathrm{~nm}$. Independently, the localization error estimated from vesicle trajectories by the method of Vestergaard et al. [35] is approximately equal to $10 \mathrm{~nm}$. The vesicle radius is determined by Hough transformation, as in the bead and droplet image analysis. The estimated uncertainty in $R$ from the standard deviation of simulated images at the appropriate SNR is approximately equal to $0.005 \mu \mathrm{m}$. More significant, however, is the standard deviation in $R$ over the course of an image series, due, for 

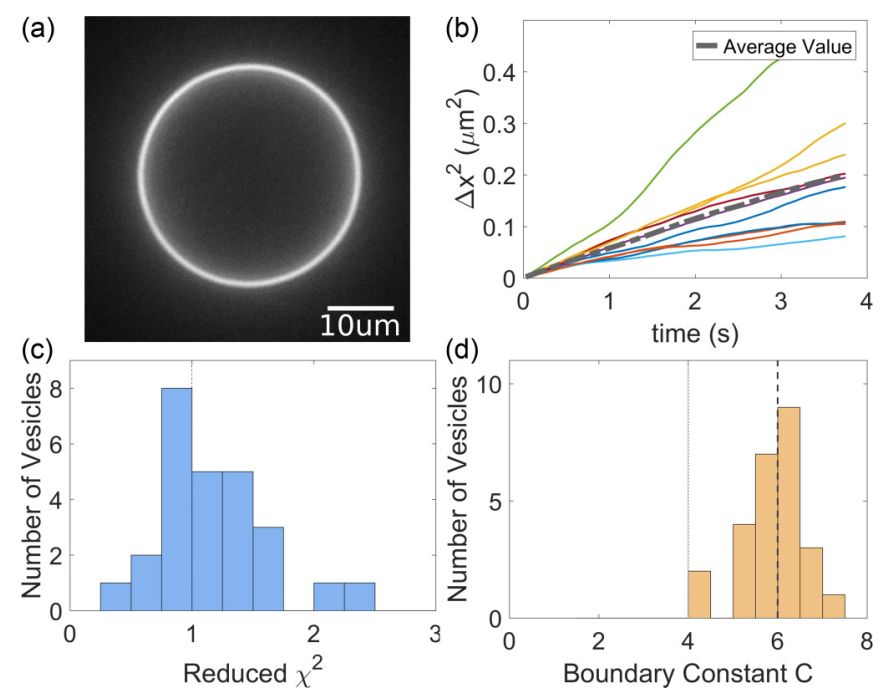

FIG. 2. (a) Typical light sheet fluorescence image of the central plane of a DOPC vesicle. (b) Mean-square displacements from ten randomly chosen vesicle trajectories (colored lines), along with their average (dashed gray line). (c) Histogram of the reduced $\chi^{2}$ values for each vesicle; $\chi^{2}=1$ indicates purely diffusive motion. (d) Histogram of $C$ for 26 vesicles giving a mean plus or minus standard error of $C=5.92 \pm 0.13$. The dashed and dotted lines indicate the theoretical values of $C=6$ and $C=4$ for the boundary conditions of a rigid sphere and an inviscid (zero shear stress) sphere, respectively.

example, to changes in position relative to the sheet plane. This is approximately equal to $0.03 \mu \mathrm{m}$, which is small compared to the typical $10 \mu \mathrm{m}$ vesicle radii and which contributes negligibly to the overall uncertainty in $C$. From vesicle position data, we calculated $D$ and $C$ as described above.

\section{RESULTS}

Light sheet fluorescence microscopy provides clear images of lipid vesicles. A typical example is shown in Fig. 2(a). Assessment of images as described above provides vesicle positions, radii, diffusion coefficients $D$, and the flow boundary constant $C$, along with their uncertainties. We provide the complete set of positions, radii, diffusion coefficients, and boundary coefficient values for every microsphere, water droplet, and lipid vesicle examined in [34].

In Fig. 2(b) we show ten randomly chosen examples of vesicle mean-square displacement (MSD) (colored lines) as a function of lag time together with their average (dashed gray line). The linearity of the MSD curve is indicative of free diffusion. A more robust assessment of the Brownian character of vesicle motion comes from the goodness of fit calculation provided by the covariance-based estimator of $D$, which gives a reduced $\chi^{2}$ value whose value should be approximately equal to 1 for a model of pure diffusion. A histogram of the measured $\chi^{2}$ values is shown in Fig. 2(c) and is consistent with simple Brownian diffusion.

As described in Sec. II, we use measurements of vesicle radii and diffusion coefficients to determine the flow boundary condition constant $C$. The histogram of $C$ for $N=26$ vesicles is shown in Fig. 2(d). The mean plus or minus standard error is $C=5.92 \pm 0.13$. To estimate possible systematic error, we assume that the polystyrene microspheres for which we calculated $C$ (see Sec. II) are ideal hard spheres. The standard deviation of the beads' $C$ value would therefore be the spread inherent in our methodology. In order to account for this uncertainty we can compute a systematic standard error by dividing the standard deviation of the beads' $C$ by the square root of the number of vesicles. This gives us a constant for vesicles of $C=5.92 \pm 0.13$ (stat.) \pm 0.16 (syst.).

\section{CONCLUSION}

Our measurements show that at least over micrometer length scales and millisecond-to-second timescales, lipid bilayers are well described by the shear stress boundary conditions that characterize solid surfaces in fluid. Perhaps reassuringly, the standard assumption that is ubiquitous in treatments of liposome hydrodynamics is well supported.

Our method illustrates that a conceptually simple imagingbased approach can provide precision measurements of microscale fluid properties. We expect that this can be extended to, for example, fluctuating membranes driven by either temperature $[30,41]$ or active forces [42] to investigate couplings between topography and drag. Put differently, the precision with which the value $C=6$ can be determined, even if one views the value itself as an unsurprising confirmation of expectations, illuminates the precision that future studies of more exotic membrane systems can achieve. Finally, we note that the methods presented here will also be applicable in non-Newtonian fluids, for which detailed understanding of microscale rheology continues to be an active area of study.

\section{ACKNOWLEDGMENTS}

This material was based in part upon work supported by the National Science Foundation under Award No. 1507115. Any opinions, findings, and conclusions or recommendations expressed in this material are those of the author(s) and do not necessarily reflect the views of the National Science Foundation.
[1] J. C. Stachowiak, D. L. Richmond, T. H. Li, A. P. Liu, S. H. Parekh, and D. A. Fletcher, Proc. Natl. Acad. Sci. USA 105, 4697 (2008).

[2] A. T. Brown, J. Kotar, and P. Cicuta, Phys. Rev. E 84, 021930 (2011).

[3] S. R. Tabaei, J. J. J. Gillissen, S. Block, F. Höök, and N. J. Cho, ACS Nano 10, 8812 (2016).
[4] M. Vögele, J. Köfinger, and G. Hummer, Phys. Rev. Lett. 120, 268104 (2018).

[5] P. Cicuta, S. L. Keller, and S. L. Veatch, J. Phys. Chem. B 111, 3328 (2007).

[6] T. T. Hormel, S. Q. Kurihara, M. K. Brennan, M. C. Wozniak, and R. Parthasarathy, Phys. Rev. Lett. 112, 188101 (2014). 
[7] T. T. Hormel, M. A. Reyer, and R. Parthasarathy, Biophys. J. 109, 732 (2015).

[8] V. L. Thoms, T. T. Hormel, M. A. Reyer, and R. Parthasarathy, Langmuir 33, 12510 (2017).

[9] S. Mendez and M. Abkarian, Phys. Rev. Fluids 3, 101101 (2018).

[10] K. D. Lee, S. Nir, and D. Papahadjopoulos, Biochemistry 32, 889 (1993).

[11] C. He, Y. Hu, L. Yin, C. Tang, and C. Yin, Biomaterials 31, 3657 (2010).

[12] J. T. Mason and C. Huang, Ann. N.Y. Acad. Sci. 308, 29 (1978).

[13] J. J. Foo, K. K. Liu, and V. Chan, AIChE J. 50, 249 (2004).

[14] S. Johnson, A. Bangham, M. Hill, and E. Korn, Biochim. Biophys. Acta 233, 820 (1971).

[15] M. Kraus, W. Wintz, U. Seifert, and R. Lipowsky, Phys. Rev. Lett. 77, 3685 (1996).

[16] R. Waugh, Biophys. J. 38, 19 (1982).

[17] A. R. Honerkamp-Smith, F. G. Woodhouse, V. Kantsler, and R. E. Goldstein, Phys. Rev. Lett. 111, 038103 (2013).

[18] F. G. Woodhouse and R. E. Goldstein, J. Fluid Mech. 705, 165 (2012).

[19] S. Reuther and A. Voigt, J. Comput. Phys. 322, 850 (2016).

[20] E. P. Petrov, R. Petrosyan, and P. Schwille, Soft Matter 8, 7552 (2012).

[21] T. H. Li, J. C. Stachowiak, and D. A. Fletcher, in Methods in Enzymology, edited by N. Duzgunes (Academic, New York, 2009), Vol. 465, pp. 75-94.

[22] S. Shkulipa, W. den Otter, and W. Briels, Biophys. J. 89, 823 (2005).

[23] B. Cross, A. Steinberger, C. Cottin-Bizonne, J. Rieu, and E. Charlaix, Europhys. Lett. 73, 390 (2006).

[24] Y. Kaizuka and J. T. Groves, Biophys. J. 86, 905 (2004).

[25] B. L. Stottrup, D. S. Stevens, and S. L. Keller, Biophys. J. 88, 269 (2005).
[26] W. M. Deen, Analysis of Transport Phenomena (Oxford University Press, Oxford, 1998).

[27] J. S. Hadamard, C. R. Acad. Sci. 152, 1735 (1911).

[28] W. Rybczynski, Bull. Acad. Sci. Cracovie Ser. A 1, 40 (1911).

[29] V. Ntziachristos, Nat. Methods 7, 603 (2010).

[30] A. F. Loftus, S. Noreng, V. L. Hsieh, and R. Parthasarathy, Langmuir 29, 14588 (2013).

[31] M. J. Taormina, M. Jemielita, W. Z. Stephens, A. R. Burns, J. V. Troll, R. Parthasarathy, and K. Guillemin, Biol. Bull. 223, 7 (2012).

[32] P. J. Keller, A. D. Schmidt, J. Wittbrodt, and E. H. Stelzer, Science 322, 1065 (2008).

[33] R. Parthasarathy, Nat. Methods 9, 724 (2012).

[34] See Supplemental Material at http://link.aps.org/supplemental/ 10.1103/PhysRevResearch.2.013132 for all data and a movie of a light sheet fluorescence microscopy scan through a single vesicle.

[35] C. L. Vestergaard, P. C. Blainey, and H. Flyvbjerg, Phys. Rev. E 89, 022726 (2014).

[36] J. Illingworth and J. Kittler, IEEE Trans. Pattern Anal. Mach. Intell. PAMI-9, 690 (1987).

[37] K. D. Chen, Y. F. Lin, and C. H. Tu, J. Chem. Eng. Data 57, 1118 (2012).

[38] J. T. Groves, R. Parthasarathy, and M. B. Forstner, Annu. Rev. Biomed. Eng. 10, 311 (2008).

[39] S. L. Veatch, in Lipid Rafts, edited by T. J. McIntosh (Humana, Totowa, 2007), pp. 59-72.

[40] J. F. Swindells, C. F. Snyder, R. C. Hardy, and P. E. Golden, Viscosities of Sucrose Solutions at Various Temperatures: Tables of Recalculated Values, Natl. Bur. Stand. Circ. 440 (U.S. GPO, Washington, DC, 1958).

[41] J. Henriksen, A. C. Rowat, and J. H. Ipsen, Eur. Biophys. J. 33, 732 (2004).

[42] J. B. Manneville, P. Bassereau, D. Lévy, and J. Prost, Phys. Rev. Lett. 82, 4356 (1999). 